\title{
CROSS-LAYER SIMULATION AND OPTIMIZATION FOR MOBILE AD-HOC NETWORKS
}

\author{
André-Luc Beylot ${ }^{1}$, Riadh Dhaou $^{1}$, Vincent Gauthier ${ }^{2}$, and Monique Becker ${ }^{2}$ \\ ${ }^{1}$ ENSEEIHT, IRIT/TeSA Lab \\ Toulouse, France \\ \{Andre-Luc.Beylot, Riadh.Dhaou\} @enseeiht.fr \\ ${ }^{2}$ GET/INT, Samovar Lab. \\ Evry, France \\ $\{$ Vincent.Gauthier, Monique.Becker\}@int-evry.fr
}

\begin{abstract}
This paper introduces cross-layer implementation with a multi-rate aware routing scheme and shows that SNR is an important information to use in a routing protocol. The existing routing protocol attempts to minimize the number of hops between source-destination pairs. We use a new metric definition to route the packets and to select the best available link along the path in a multi-rate protocol senario. The new metric is created with information coming from interlayer interaction between the routing layer and the MAC layer. We use SNR as an information about link quality. We show through simulation that for communications using muti-rate protocol in ad hoc networks, throughput is highly affected as soon as the route goes through low-rate link.
\end{abstract}

Keywords: Ad hoc networks, AODV, Cross-Layers, IEEE 802.11, Inter-layer interactions, Performance, QoS

\section{Introduction}

Ad hoc wireless network are self organizing multi-hop wireless networks where all the nodes take part in the process of forwarding packets. Ad Hoc networks are very different from conventional computer networks. First, the radio resource is rare and time varying. Second, the network topology is mobile and the connectivity is unpredictable. Third architecture-based 802.11 WLAN, is further complicated due to the presence of hidden stations, exposed station, "capturing" phenomena, and so on. Fourth, many current and proposed wireless networking standards have this multi-rate capacity $(802.11 \mathrm{~b}, 802.11 \mathrm{a}$, $802.11 \mathrm{~g}$, and HyperLan2). The interaction between these phenomena make the behavior of ad hoc network very complex to predict and are really different 
from wired network architecture.

The aim of Cross-Layer concept is to improve the performance of all layers and share key information between these layers. The goal of this technique is to take benefit of informations about the channel quality to develop a more powerful routing technique. The inter-layer interaction will be managed by the network status. The network status will act as information repository and it will give on demand to each layer, the information about other layers. The inter-layer interaction enables us to use the information on the channel to define a new cost metric in ad hoc network as a function of link quality.

Our proposed schemes use a cross-layer interaction between MAC and network layer. The objective is to create a new QoS cost metric (cf Fig. 1). The proposed metric is a function of SNR (Signal Noise Ratio) and of the number of hops.

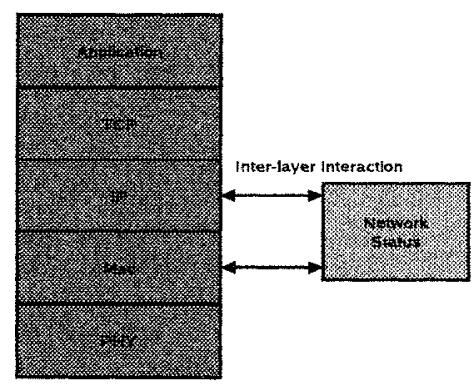

Figure 1. Inter-Layer Interaction

\section{Related Work}

In [BAR04] the authors proposed a new network metric the medium Time Metric (MTM), which is derived from a general theoretical model of the reachable throughput in multi-rate ad hoc wireless networks. The MTM avoids using the long range link favored by shortest path routing in favor of shorter, higher throughput, more reliable links.

In [GCNB03] the authors propose a new power-aware routing technique for wireless ad hoc networks (PARO) where all nodes are located within the maximum transmission range of each other. PARO uses a packet forwarding technique where immediate nodes can elect to be redirector on behalf of sourcedestination pairs with the goal of reducing the overall transmission power needed to deliver packet in the network, thus, increasing the operational lifetime of network devices.

In [DACM02] the authors show that the minimum hop path generally contains 
links which exhibit low reliability. In [DRWT97] and [HLT02] the authors present routing protocols which are based on signal stability rather then on only a shortest path in order to provide increased path reliability.

Based on the IEEE 802.11 protocol, the Receiver Based Auto Rate (RBAR) protocol was presented in [GHB01]. RBAR allows the receiving node to select the rate. This is accomplished by using the SNR or the RTS packet to choose the most appropriate rate and to communicate that rate to the sender using the CTS packets. This allows much faster adaptation to the changing channel conditions than ARF, but requires some modifications to the 802.11 standard.

The Opportunistic Auto Rate (OAR) protocol which is presented in [BSK02], operates using the same receiver based approach, but allows high-rate multipacket burst to take advantage of the coherence time of good channel conditions. The bursts also dramatically reduce the overhead at high rates by smoothing the cost of contention period and RTS CTS frames over several packets.

\section{IEEE 802.11 MAC Layer Approach}

The IEEE 802.11 technology is a good platform to implement single-hop ad hoc network because of its extreme simplicity. But in a multi-hop ad hoc networks environment, the IEEE 802.11 protocol works inefficiently. There are two main effects that reduce the *effiency* of the protocol. First the $802.11 \mathrm{~b}$ standard extends the 802.11 standard by introducing a higher-speed Physical Layer in the $2.4 \mathrm{Ghz}$ band still guaranteeing the interoperability with 802.11 cards. The $802.11 \mathrm{~b}$ standard enables multi-rate transmission at $11 \mathrm{Mbps}$ and $5.5 \mathrm{Mbps}$ in addition to $1 \mathrm{Mbps}$ and $2 \mathrm{Mbps}$. To ensure the interoperability, each WLAN defines a basic rate set that contains the data transfer rate that must be used by all the stations in a WLAN. The overhead due to the use of the basic rate between all the stations in a WLAN is very important and it affects the throughput.

- $T_{c t r}$ is the time required to transmit all the control frame $T_{c t r}=T_{r t s}+$ $T_{c t s}+T_{a c k}$.

- $T_{a c k}$ is the time required to transmit MAC ACK frame which includes Physical header and MAC header.

- $T_{\text {data }}$ is the time required to transmit a MAC data frame which includes Physical header, MAC header, MAC payload

- $T_{s t}$ is the Slot Time.

- $T_{\text {payload }}$ is the time required to transmit only the $m$ bytes generated by the application; $T_{\text {payload }}$ is therefore equal to $m /$ data rate where 


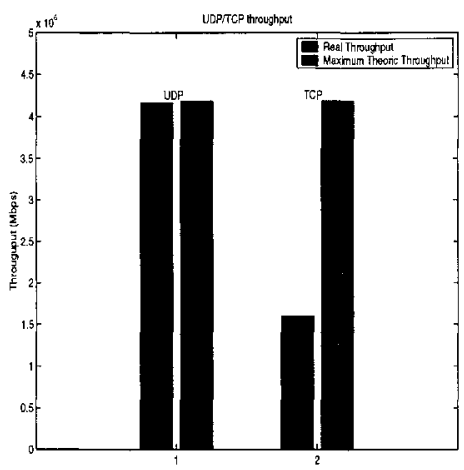

Figure 2. real throughput vs theoretic throughput with constant size packet of 1024

data rate is the data rate used by the NIC to transmit data, i.e., $1,2,5.5$ or $11 \mathrm{Mbps}$.

- $\frac{C W \min }{2} * T_{s t}$ is the average backoff time

$$
T h=\frac{T_{\text {payload }}}{T_{\text {difs }}+T_{c t r}+T_{\text {data }}+3 * T_{\text {sifs }}+\frac{C W \min }{2} * T_{s t}}
$$

However, even with large packet size (eg., $m=1024$ bytes) the bandwidth utilization is lower than $39 \%$ (cf Fig. 2). This theoretical analysis corresponds to the measurement of the actual throughput at the application level. Two typical "applications" have been considered: FTP and CBR. The experimental results related to the UDP traffic are very close to the maximum throughput computed analytically. As expected, in the presence of TCP traffic the measured throughput is lower than the theoretical maximum throughput. Indeed, when using the TCP protocol overhead related to the TCP-ACK transmission has to be taken into account.

In the second graph (cf Fig. 3), qualnet simulations have been run for which one "CBR" application have been considered (packets size $=1024$ bytes). The throughput has been studied as a function of the number of hops and for different available rate using the IEEE $802.11 \mathrm{~b}$ protocol. We could see how the transmission throughput decreases as a function of the number of hops.

\section{Effect of the SNR}

Many current and proposed wireless network standards such as IEEE 802.11a, IEEE 802.11 b or HyperLan 2 present a multi-rate capacity. The IEEE802.11b has different adaptive modulations which were investigated with the dynamic 


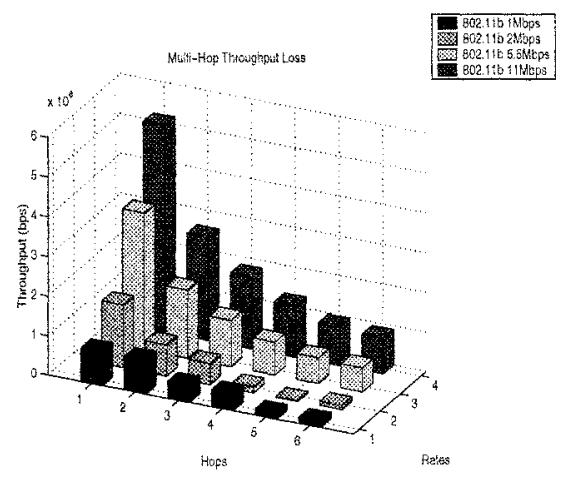

Figure 3. Throughput vs number of hop

channel allocation technology. All of them are trying to improve the effective data rate given the specified bit error rate (BER). Due to the physical properties of communication channels, there is a direct relationship between the rate of communication and the quality of the channel required to support that communication reliably. Since the distance is one of the primary factor that determines wireless channel quality, there is an inherent trade-off between high transmission rate and effective transmission range. The SNR is a very interesting information to monitor because it reflects the link quality. In a multi-rate protocol, each available link may operate at a different rate. The most important challenge is to choose a good trade-off between the link quality and the number of hops. As a short link can operate at high rate, more hops are required to reach the destination.

The Figure 4 and 5 show the Bit Error rate is represented as a function of SNR and the Throughput as a function of the SNR (cf Fig. 5). We can see the effect of the SNR on the transmission performance. But the distance of each link is the primary factor that determines channel quality. Long links have low quality, and thus operate at low rate (cf Fig. 4). Nevertheless, it is difficult to measure the link quality, so we propose to use the Smoothed value of Signal-to-Noise-Ratio since SNR could change dynamically with a high frequency due to electro-magnetic effect. This Smoothed SNR (SSNR) value can be computed as follows:

$$
\operatorname{ssn} r=(1-\alpha) * \text { old_snr }+\alpha * \text { cur_sn } r
$$

where $c u r_{-} s n r$ and old_snr denote the value of the SNR on receipt of a packet and the previously computed ssn $r$, respectively. The constant value $\alpha$ is a filtering factor and it is set between 0.7 and 0.9 as a function of changing speed of the signal. 


\begin{tabular}{|c|c|c|c|c|}
\hline Range(meters) & $11 \mathrm{Mbps}$ & $5.5 \mathrm{Mbps}$ & $2 \mathrm{Mbps}$ & $1 \mathrm{Mbps}$ \\
\hline Open & $160 \mathrm{~m}$ & $270 \mathrm{~m}$ & $400 \mathrm{~m}$ & $550 \mathrm{~m}$ \\
\hline Semi-open & $50 \mathrm{~m}$ & $70 \mathrm{~m}$ & $90 \mathrm{~m}$ & $115 \mathrm{~m}$ \\
\hline Closed & $25 \mathrm{~m}$ & $35 \mathrm{~m}$ & $40 \mathrm{~m}$ & $50 \mathrm{~m}$ \\
\hline
\end{tabular}

Figure 4. Relation between the distance of two nodes and the available data rate

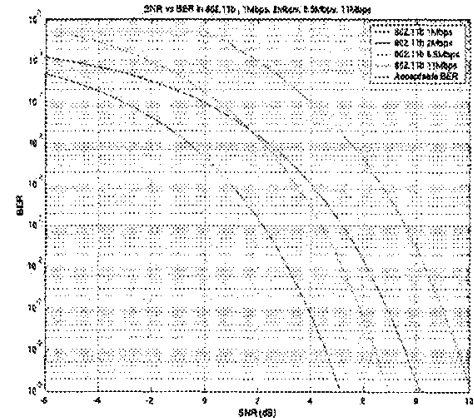

Figure 5. Bits error rate as a function of SNR

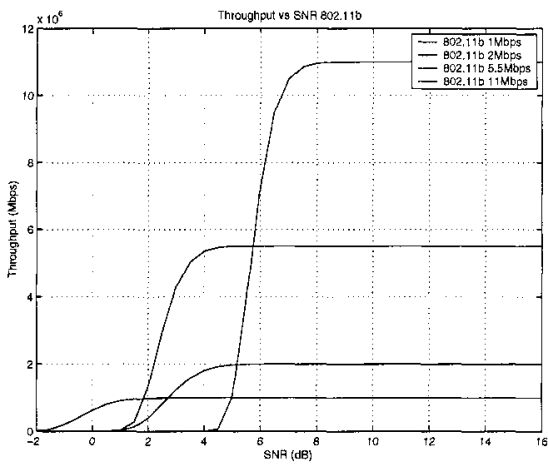

Figure 6. Throughput as a function of SNR

\section{Network Layer Approach}

Ad-Hoc networks require a highly adaptive routing scheme to deal with the frequent topology changes and low performance. In this paper, we propose a routing protocol that utilizes the ad hoc network characteristics to select the route which has a better compromize between the number of hops, the theoretical available bandwidth, and the stability of the route. This protocol is new because it uses the signal strength and SNR available at the MAC layer of an individual host as a route selection criteria. The trade-off between the number of hops and the SNR of each individual route defines a new network metric. The new metric available at the network layer allows to have a global overview of the best available path. In this protocol, a host initiates route discovery ondemand (only when a route is needed to send data). The source broadcasts a route-search packet which will be propagated to the destination, allowing the destination to choose a route and return a route reply.

This paper describes an implementation of the AODV protocol based on a cross layer mechanism in which we use SNR information to obtain better routing techniques. To do so, we use SNR of each node to determine the route which will have globally the best SNR along the path. For this purpose we have added SNR information in each RREQ packet which is used as QoS in- 


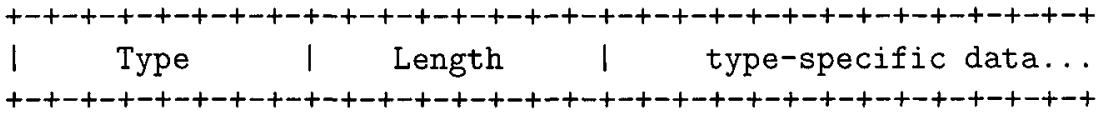

Figure 7. Cross-Layer Extension in AODV Frame

formation. Each node that forwards these packets adds its own SNR information, thus updating the SNR of each link along the route. When the destination node receives the RREQ packet it directly has the information about the quality of the route. The destination node then determines the best route and replies by sending a RREP packet so that each node on the route can save the QoS information in its routing table. With a route decision mechanism, we take advantage of this QoS information, the route quality and the global throughput are improved.

The RREQ and the RREP AODV frame carry the new extension field (cf Fig. 7). Each host along the path picks back the new metric in the extension field of the RREQ frame. In the case of RREP frame, each host along the path reads the new metric value and stores it in its routing table.

As we always find the link with the best SNR, we obtain a path with small transmission range but it may increase the number of hops. Consequently, the channel access overhead (e.g, backoff time) could be increased in proportion with the hop count. However it can reduce the link-level transmission time ( $\simeq$ Packet Size / Bandwidth), which is highly affected by the packet size. By reducing the transmission time, we can achieve a better throughput and always reduce the total energy consumption in the network wide.

This protocol have been tested with ns- 2 and is available. It is developed with the source code of AODV-UU[LN] with cross-layer extension[Gau04].

\section{Expected Result}

In the first part of this paper we have presented different effects of SNR on link quality. First, the throughput and the SNR are directly correlated to the distance between the sender and the receiver (cf Fig. 9). Second, the throughput is directly correlated to the SNR (cf Fig. 8).

A good link quality is defined by a good SNR. The SNR is a good indicator of the quality of service of the link. But it is more convenient to aggregate all SNR informations available on each link into one metric indicator for each path.

We chose this new metric to find the best available path and to globally improve the network performance. Each link of the best path will have a low Bit 


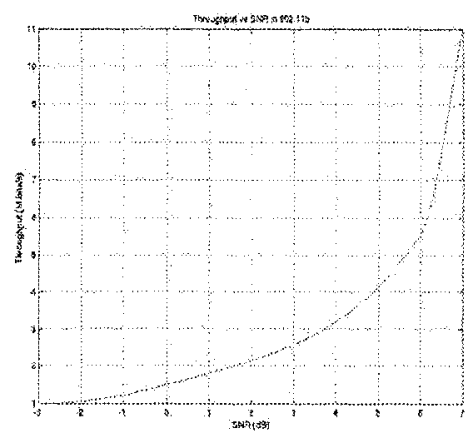

Figure 8. Throughput vs SNR

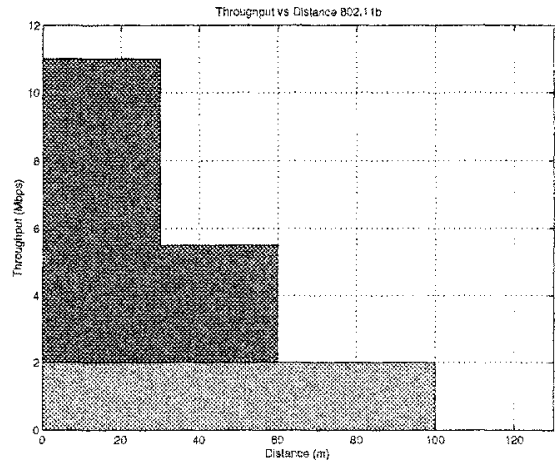

Figure 9. Distance vs Throughput in $802.11 \mathrm{~b}$

Error Rate and the selected path has the best available throughput between the sender and the receiver. The measuring method of SNR helps us to have a good overview over the time of the SNR information and not just the SNR at a given selected time. It helps us to determine which link has a good stability over the time and which link has the lower probability to shutdown.

\section{Future Works}

The new challenge is to develop a mechanism to monitor the link quality in real-time during the communication. In this case when the quality of service of a link falls down, a mechanism should be implemented to find a new path. It will also be necessary to propose a trade-off between the number of hops and the quality of each link. It will not lead to select the best available route but to the selection of a route which presents the best compromise between the number of hops and the available data rate.

This mechanism could help us to develop a new method of load-balancing because each node could monitor the number of path search demands and determine a trade-off not to be crossed.

\section{Simulation Issues}

We run simulations in order to test the performance and to validate crosslayer protocols We have written modules simulating a layered stack, which includes MAC layer and network layer. Each layer module communicates with the upper and the lower layer modules and each layer module communicates also with the other modules of the same layer in other stacks (see Fig. 1). Modules inside the stack are supposed to communicate with the cross-layer stack. 
To simulate these communications, a design solution has been implemented. Small experience maybe hand maneged but for longer experiments, simulation should have to be automatically and dynamically run. So it is necessary to design dynamic and autonomic simulations, which are not easy. This part of the work is not completed yet.

\section{Conclusion}

In this paper, we started to investigate several cross-layer protocols and we have presented implementation of the AODV protocol which includes crosslayer extension. The concept of cross-layer provides a wide field of information exchange between layers. We focused on SNR which is a useful information to exchange because a low SNR level impacts throughput on the path. A low SNR level leads to a high bit error rate and consequently to a low link throughput.

This protocol uses SNR information in the calculating of the network metric to choose the link with the best available quality (low bit error rate and high throughput). In wireless networks major criteria are the radio channel quality and the energy consumption. These elements cannot be only managed at a local level but have to be managed in a distributed way in the network. Consequently a new network metric has to be created, instead of looking only for the number of hops between the transmitter and the receiver. The quality of the radio channel along the route will also considered.

In order to validate cross-layer methods it is necessary to run a lot of very long simulations in an autonomic and a dynamic way. This work is not completed yet. Nevertheless, cross-layer will probably lead to get very useful results about ad-hoc networks optimization.

The design of dynamic and autonomic simulations may be of general use in order to solve a large set of problems.

\section{References}

[BAR04] D. Holmer B. Awerbuch and H. Rubens. High throughput route selection in multi-rate ad hoc wireless networks. In proceedings of Wireless On-demand Network Systems WONS 2004, Madonna di Campiglio, Italy, 2004.

[BBD03] M. Becker, AL. Beylot, and R. Dhaou. Aggregation methods for performance evaluation of communication networks, performance evaluation. In proceedings of International Symposium of Performance evaluation - Stories and Perspectives, Vienna, Austria, Eds: G. Kotsis, pp 215-230, 2003.

[BSK02] A. Sabharwal B. Sadeghi, V. Kanodia and E. Knightly. Opportunistic media access for multirate ad hoc networks. In proceedings of ACM MOBICOM 2002, Atlanta, GA, 2002.

[DACM02] D. De Couto, D. Aguayo, B. Chambers, and R. Morris. Performance of multihop wireless networks: Shortest path is not enough. In The First Workshop on 
Hot Topics in Networks (HotNets-I), Princeton, New Jersey, October 2002. In proceedings of ACM SIGCOMM.

[DRWT97] R. Dube, C. Rais, K. Wang, and S. Tripathi. Signal stability based adaptive routing (ssa) for ad hoc mobile networks. IEEE Personal Communication, 1997.

[GAG03] M. Conti G. Anastasi, E. Borgia and E. Gregori. IEEE 802.11 ad hoc networks: Performance measurements. In proceedings of Distributed Computing Systems Workshops ICDCSW'03, Rhode Island, USA, May 2003.

[Gau04] Vincent Gauthier. Adov-uu with cross-layer, 2004. French National Institute of Telecommuncation, http://www-rst.int-evry.fr/ gauthier/.

[GCNB03] J. Gomez, A .Campbell, M. Naghshineh, and C. Bisdikian. Paro: Supporting dynamic power controlled routing in wireless ad hoc networks. ACM/Kluwer Journal on Wireless Networks (WINET), 9(5), 2003.

[GHB01] N. H. Vaidya G. Holland and P. Bahl. A rate-adaptative mac protocol for multihop wireless networks. In proceedings of Mobile Computing and networking, 2001.

[GK99] P. Gupta and P. Kumar. Capacity of wireless networks. Technical report, University of Illinois, Urbana-Champaign, 1999.

[GW02] A. J. Goldsmith and S. Wicker. Design challenges for energy-constraind ad hoc wireless networks. IEEE Wireless Communication, 2002.

[HLT02] E. Nordstrom H. Lundgren and C. Tschudin. Coping with communication gray zone in IEEE based ad hoc networks. In proceedings of WoWMoM, 2002.

[KH95] R. Knopp and P. A. Humblet. Information capacity and power control in singlecell multiuser communication. In proceedings of IEEE International Communications Conference. Seattle, 1995.

[KK03] V. Kawadia and P. R. Kumar. A cautionary perspective on cross layer design. Submitted to IEEE Wireless Communication Magazine, July 2003.

[LN] Henrik Lundgren and Erik Nordstrem. Adov-uu. Uppsala University, http://user.it.uu.se/ henrikl/aodv/.

[MBTB98] M. Moulki, AL. Beylot, L. Truffet, and M. Becker. An aggregation technique to evaluate the performance of a two-stage buffered atm switch. In Annals of Operations Research 79, pp 373-392, Baltzer, 1998.

[PR99] C. E. Perkins and E. Royer. Ad-hoc on demand distance vector routing. In proceedings of 2nd IEEE Workshop Mobile Comp. Sys. and Apps. (WMCA), New Orleans, LA, Feb 1999.

[PRDM00] C. E. Perkins, E. Royer, S. Das, and M. Marina. Performance comparaison of two on-demand routing protocol for ad hoc networks. In proceedings of IEEE INFOCOM, 2000.

[SATT04] M. Saito, H. Aida, Y. Tobe, and H. Tokuda. proximity-based dynamic path shortening scheme for ubiquitous ad hoc. In proceedings of Distributed Computing Systems, 2004.

[SS03] J. Chen S. Sheu, Y. Tsai. Mr2rp: The multi-rate and multi-range routing protocol for ieee 802.11 ad hoc wireless networks. Wireless Networks The Journal of Mobile Communication, Computation and Information, Volume 9(Issue 2), 2003.

[YSC03] J. Park Y. Seok and Y. Choi. Multi-rate aware routing protocol for mobile ad hoc networks. IEEE VTC 2003-Spring, Jeju, Korea, 2003. 